\title{
Optimization of transport vehicle transmission vibroacoustic characteristics
}

\author{
Alexander Taratorkin ${ }^{1,}$ Victor Derzhanski1 ${ }^{1,2}$, and Igor Taratorkin ${ }^{1, *}$ \\ ${ }^{1}$ IES UB RAS, Department of Transportation Vehicles Mechanics, 620049, Ekaterinburg, Russian Federation \\ ${ }^{2}$ Kurgan State University, Department of Applied Mechanics, 640020, Kurgan, Russian Federation
}

\begin{abstract}
One of the main sources of vibroacoustic emissions is the transmission of a transport vehicle. Providing a high level of fuel economy performance and an acceptable level of power-train noise and vibration $(\mathrm{NVH})$ is a complex engineering task and a real professional challenge for NVH specialists. The purpose of the research is to provide the required level of vibroacoustic characteristics of the transport vehicle transmission being developed at the design stage through a feasible correction of structural characteristics. To achieve this goal requires deeper understanding the dynamic phenomena of $\mathrm{NVH}$, as well as expansion of the virtual design methods. We used multiphase analytical methods that allow us to estimate operation dynamics and vibroacoustic response of the transmission. Based on application of advanced CAE methods, a structural analysis and subsequent calculation of acoustic radiation is performed using the example of an automatic transmission (AT) prototype which is being developed for a vehicle of category N. The ability to control acoustic emissions is demonstrated by adjusting the structural (modal) parameters of the transmission case. Engineering solutions (configuration modifications) are being worked out to change the structural state (Limit state design) of the AT configuration (structural optimization) to ensure an acceptable level of acoustic emissions. For the gearbox in question, the first 50 waveforms (mode shapes, wave mode)were identified in the frequency range up to $8 \mathrm{kHz}$. The analysis made it possible to conclude that the greatest contribution to the integral noise level is made by wave forms at a frequency of $950 \mathrm{~Hz}$, the internal partitions of the gearbox housing; at a frequency of $1324 \mathrm{~Hz}$, the side walls of the shaft part of the gearbox and at a frequency of $1439 \mathrm{~Hz}$ are connected oscillations of the side walls and partitions between the shaft part of the gearbox and the demultiplier. To suppress these vibrations, additional finning of the partition between the shaft part of the automatic gearbox and the demultiplier was installed, as well diagonal reinforcing ribs were introduced into the design of the automatic gearbox side walls and the distributor cap. The performed measures allowed significantly reducing the integral level of acoustic pressure.
\end{abstract}

\section{Introduction}

Currently, special attention is paid to vibroacoustic behavior of the transport vehicles assembled units, that is a key factor in assessing by consumers the quality not only of VIP - class vehicles, but also general and special purpose vehicles, of various types of agricultural machinery and road construction machines. In order to ensure optimal vibroacoustic behavior of the transport vehicle transmission, it is important to focus on noise and vibration at the development stage of its design. Specific targets, such as eigen frequencies, vibration levels and noise emissions, should be determined at the initial stages of product design and continuously monitored throughout the development process. Virtual forecasting methods application focused on vibroacoustic vehicle behavior, which is formed, in particular, by the transmission, is a prerequisite for efficient costs and time expenditures, and it allows for avoiding the need to eliminate vibroacoustic problems in the process of integrating the transmission into the vehicle.

Transmissions are the ones being the main sources of noise and vibration in vehicles. Under certain operating conditions, the noise emitted by the transmission may dominate both in the internal and external noise levels, and under some conditions, the vibration generated by the transmission prevails in the overall level of vibration perceived by the driver.

The factors that form the vibroacoustic activity of the transmission are determined by the structural parameters of the gears and the modal characteristics of the transmission casing, which interact with each other through bearing supports. At the same time, the noise emitted by the transmission is one of the main sources that leads to a violation of ergonomic requirements, both for the driver and for the environment. In accordance with the standard classification, the acoustic radiation of gearboxes, transmission is divided into three types, i.e. gearwhine, rattle and clunk.

\footnotetext{
Corresponding author alexandr_tar@mail.ru
} 
Rattle and clunk results from dynamic system imperfections, manifested in resonance excitation or from control system imperfections, also leading to their occurrence. These phenomena have been studied by many researchers, including the authors. The study of oscillations in the pre-torque-converter zone of the hydromechanical transmission, the conditions for exciting resonances in a nonlinear system, and development of the methods for their damping are given in [1]. The methods for eliminating dynamic loads using nonlinear energy absorbers (Nonlinear Energy Sinks (NESs)) in meshing of gear teeth to eliminate vibrations are described in [2].

Gearwhine results from imperfections of gear geometry and their supporting components. This type of radiation is one of the top priority manifestations of the NVH phenomenon and is characterized by the complexity of developing constructive and technological measures for its elimination [3-4]. This leads to the fact that the vehicle engineers have to rely on acoustic radiation testing, which requires a significant investment of time and money. At the same time, the mathematical and software tools describing this phenomenon is constantly being improved. A detailed study of the effects of microgeometry changes in meshing of gear teeth was studied in [4]. To forecast gear wheel dynamics and transmission errors, gear engagement designs are simulated based on the dynamics of several rigid bodies, and analyses are carried out along with gear wheel testing at the testing bench.

It is concluded that imperfection of the geometric parameters of the lateral surface of the tooth is the main prerequisite for gearwhine occurrence. In [5], the simulations are performed to decrease the noise of the reduction gear by means of transmission error reduction by modifying the gear profile. Many other works include experimental and analytical studies that attempt to reduce the level of excitation.

However, only few researchers suggest reducing the response level of a dynamic system (the transmission case) to excitation. At the same time, the acoustic radiation of the transmission arises directly on its vibrating surfaces, which exercises a function of membranes that transmit the effects of internal forces as an audible sound. These forces occur when transmitting torsional and bending moments in meshing of gear teeth, radial and axial forces in bearing supports in steady and transient modes of operation, as well as in the event of resonance excitation. Transmission vibration is directly transmitted to the vehicle structure. At the same time, it can become obvious and tangible in the form of tactile sensations on various elements of the vehicle structure (steering wheel, seats, glazing, etc.).

Thus, the goal of this research is to provide the required level of vibroacoustic characteristics of the transport vehicle transmission under development at the design stage through a feasible correction of structural parameters. This will increase the attractiveness of this transmission as a base of power-train units for various vehicles.

\section{Noise standards}

Compliance of the acoustic characteristics of mobile machines with sanitary standards is one of the most important professional safety criteria. Compliance with the requirements of standards for acoustic characteristics creates normal conditions for personnel vital activity. When assessing the noise level of vehicles, it should be considered that when the noise level is $\mathrm{La}=40 \ldots 60$ $\mathrm{dBA}$, there is a decrease in concentration, and the impact on the human nervous system increases. When the noise level is $\mathrm{La}=60 \ldots 85 \mathrm{dBA}$, the physiological stress of workers increases, visual acuity decreases, headaches and dizziness are observed. An increase in noise above $90 \mathrm{dBA}$ leads to a decrease in hearing sensitivity, an increase in intracranial and blood pressure, impaired digestion, body metabolism and the size of internal organs. The regulatory documents governing acoustic characteristics are the sanitary norms SN 2.2.4/2.1.8.562-96 and the interstate standard GOST 33555-2015. These documents regulate the levels of sound pressure in the octave frequency bands and, as an integral characteristic, the equivalent level of noise (sound emission). The maximum permissible sound limit in the workplace for category $\mathrm{N}$ vehicles is $82 \mathrm{dBA}$. Taking into account the noise attenuation at the location of the workplace in the vehicle at a distance of $1.5 \quad \ldots 2.0$ $\mathrm{m}$ from the transmission, the technical specifications for the development of transmissions set the permissible sound limit within the range of $85 \mathrm{dBA}$ and above. It should be noted that this lower limit meets the most stringent requirements in world practice.

\section{The strategy of theoretical optimization of NVH parameters}

The acoustic radiation of a transmission arises directly on its vibrating surfaces, which function as membranes that transmit the effects of internal forces as audible noise. These forces arise during torsional and bending moments transfer in gear-tooth system, radial and axial forces in bearing supports in steady-state and transient operating modes, as well as during resonance occurrence. Transmission vibration is directly transferred to the vehicle configuration.

The process of noise and vibration formation and emission can be represented in the form of a flow-chart presented in Fig. 1. To achieve the goal of the research in accordance with the glow-chart, the strategy of modeling (optimization) of $\mathrm{NVH}$ parameters of the transmission under development is used. The strategy is implemented in the LMS Virtual.Lab software package [6-12], which is a comprehensive set of software products for 3D finite element analysis and multiple design of objects, for modeling and optimization of mechanical systems while making the structural analysis of vibroacoustics characteristics. 


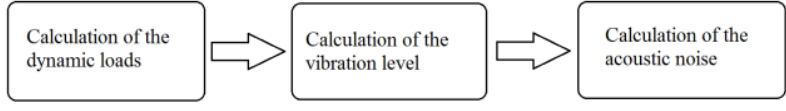

Fig. 1. Flow-chart of noise and vibration formation and emission.

The calculation was carried out in three stages:

1) Calculation of dynamic loads transmitted to the transmission case through bearing supports. A variable reaction (response variable) in gearing was adopted as the main source of loading.

2) Calculation of the vibration level of the transmission body based on the results of modal analysis and identification of the paths of vibration emission

3) Calculation of acoustic noise. Vibrational oscillations of the transmission body walls, calculated at the previous stage, are taken as the main source of external noise.

\section{Dynamic loads and vibrations calculation in the time domain}

Calculation of the dynamic loads transferred to the transmission case was carried out on the basis of the CAD model (Fig. 2), taking into account the loading conditions of the object under research. At the first stage, all the bodies of the CAD model were assumed to be absolutely rigid with mass-inertial characteristics, calculated from the geometrical characteristics of the body for steel.

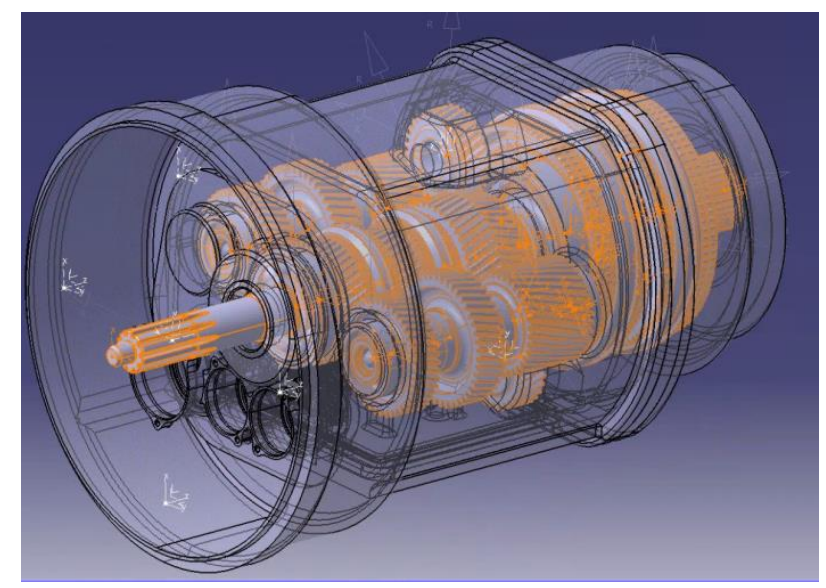

Fig.2. CAD-model of the transmission under study.

Bearing supports are modeled by ideal kinematic connections, allowing one degree of freedom. Spline joints and closed synchronizers are modeled by seals (plugs) that fully connect linear and angular displacements. External loads on the gearbox were modeled by variable values of the input shaft rotational speed $n_{\text {in }}$ and variable torque values on the output shaft $T$. The input shaft rotational speed $n_{i n}$ step by step varies from $600 \mathrm{rpm}$ to $2,200 \mathrm{rpm}$ in increments of 100 rpm.

The amount of torque moment on the output shaft considers the gear ratio on the selected gear and the variable component of the 3rd harmonic, and is calculated by the formula:

$$
T=\left(T_{\text {mean }}+T_{3} * \sin \left(3 * \omega_{\text {in }}\right)\right) * U_{i}
$$

where $T_{\text {mean }}$ is the average value of torque on the input shaft for a given mode of operation; $T_{3}$ is the amplitude of torque oscillations at the $3 \mathrm{rd}$ harmonic; $U_{i}$ - gear ratio on the selected gear; $\omega_{i n^{-}}$angular velocity $[\mathrm{rad} / \mathrm{s}]$.

The stiffness of the gearing is described by the stiffness and damping parameters (see Fig. 3), for calculation of which the theory described in [7] was used.

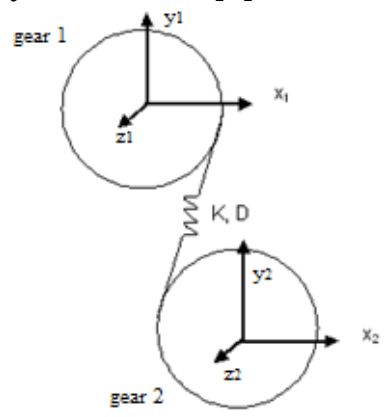

Fig. 3. Scheme for determining the elastic-dissipative characteristics in gearing.

The value of the stiffness of gearing at the point of contact corresponding to the reference diameter is calculated by the formula:

$$
k_{p}=f\left(b, H, \beta, Z_{1}, Z_{2}, X_{1}, X_{1}\right)
$$

where $b$ is the width of the tooth; $H$ is the height of the tooth; $\beta$ is the helix angle of the teeth; $Z_{1}, Z_{1}$ is the number of teeth; $X_{1}, X_{2}$ is the displacement of the original contour [7]. [7]:

Variable stiffness of gear is calculated by the formula

$$
\begin{aligned}
& k(t)=k_{p} * \exp \left[0.322 *(\beta-5)+\left(0.23 *\left(\frac{b}{H}\right)-\right.\right. \\
& \left.23.26) *\left|\frac{t-\frac{\approx * t_{z}}{2}}{1.125 * \varepsilon_{\alpha} * t_{z}}\right|^{3}\right]
\end{aligned}
$$

where $\varepsilon$ is the total contact ratio $\left(\varepsilon=\varepsilon_{\alpha}+\varepsilon_{\beta}\right)$; $\varepsilon_{\alpha}$ is transverse contact ratio; $\varepsilon_{\beta}$ is overlap ratio; $t_{z}$ is travel time of tooth $t_{z}=\frac{60}{n * z 1} ; \mathrm{n}$ is the gear rotation speed [rpm].

Based on the existing geometry, a finite element model of the hull is developed. The characteristic dimension of the element is $15 \mathrm{~mm}$, which, when using elements of the second order TET10, allows calculating local forms of wall oscillations with sufficient accuracy. The calculation of the first 50 of eigen forms shapes) and frequencies of body oscillations is performed. To take into account the static compliance of the body, the calculation of static body shapes (forms) for mounting points and bearing surface areas has been performed. The total set of forms (shapes) is orthogonalized.

Load transfer from the shafts to the body is carried out through elastic coupling, imitating the compliance of bearings. The stiffness of the supports is the same for all 


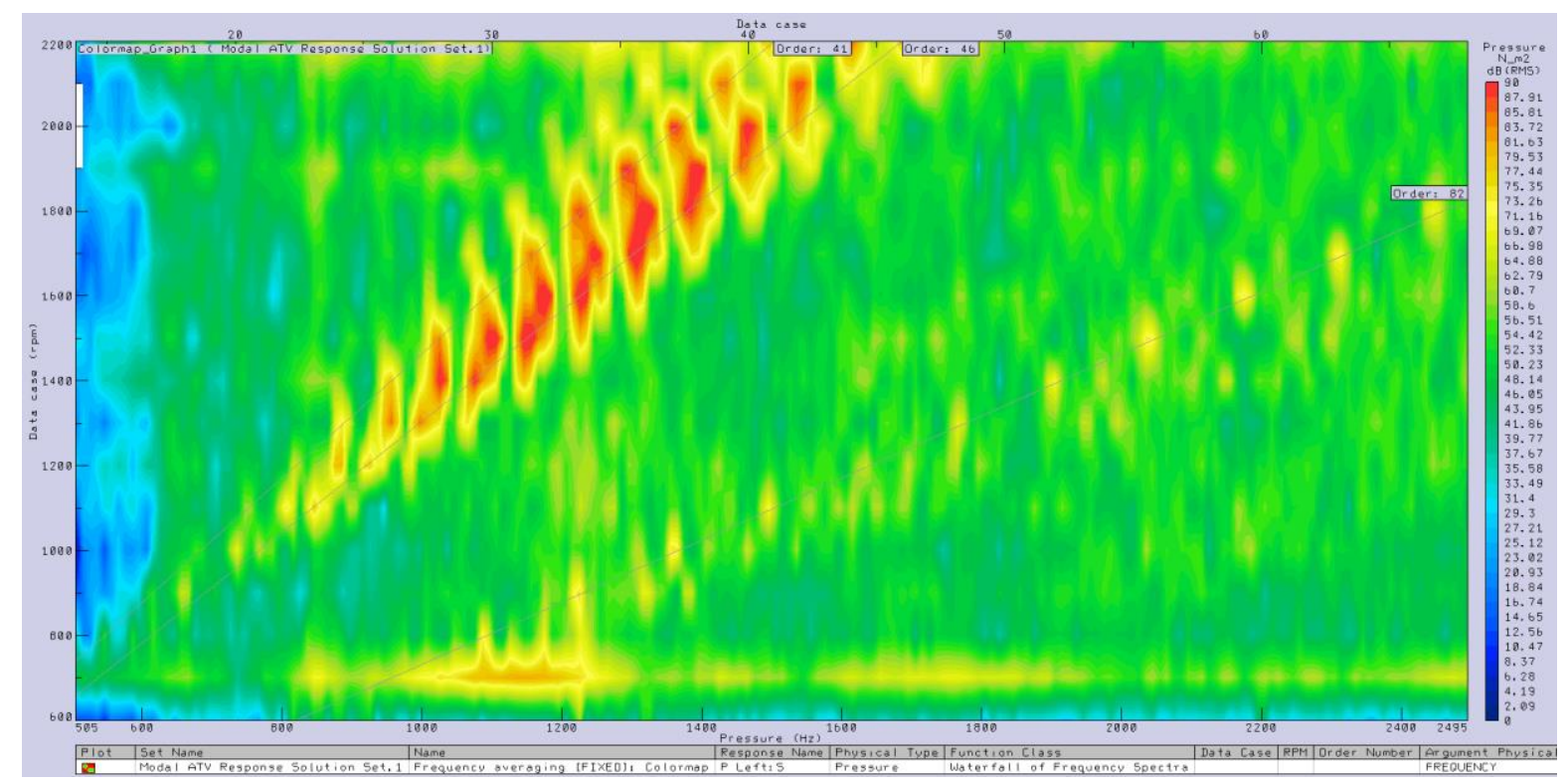

a) Dependence of the acoustic response at the desired points for each frequency of rotation of the input shaft (waterfall of frequency spectra).

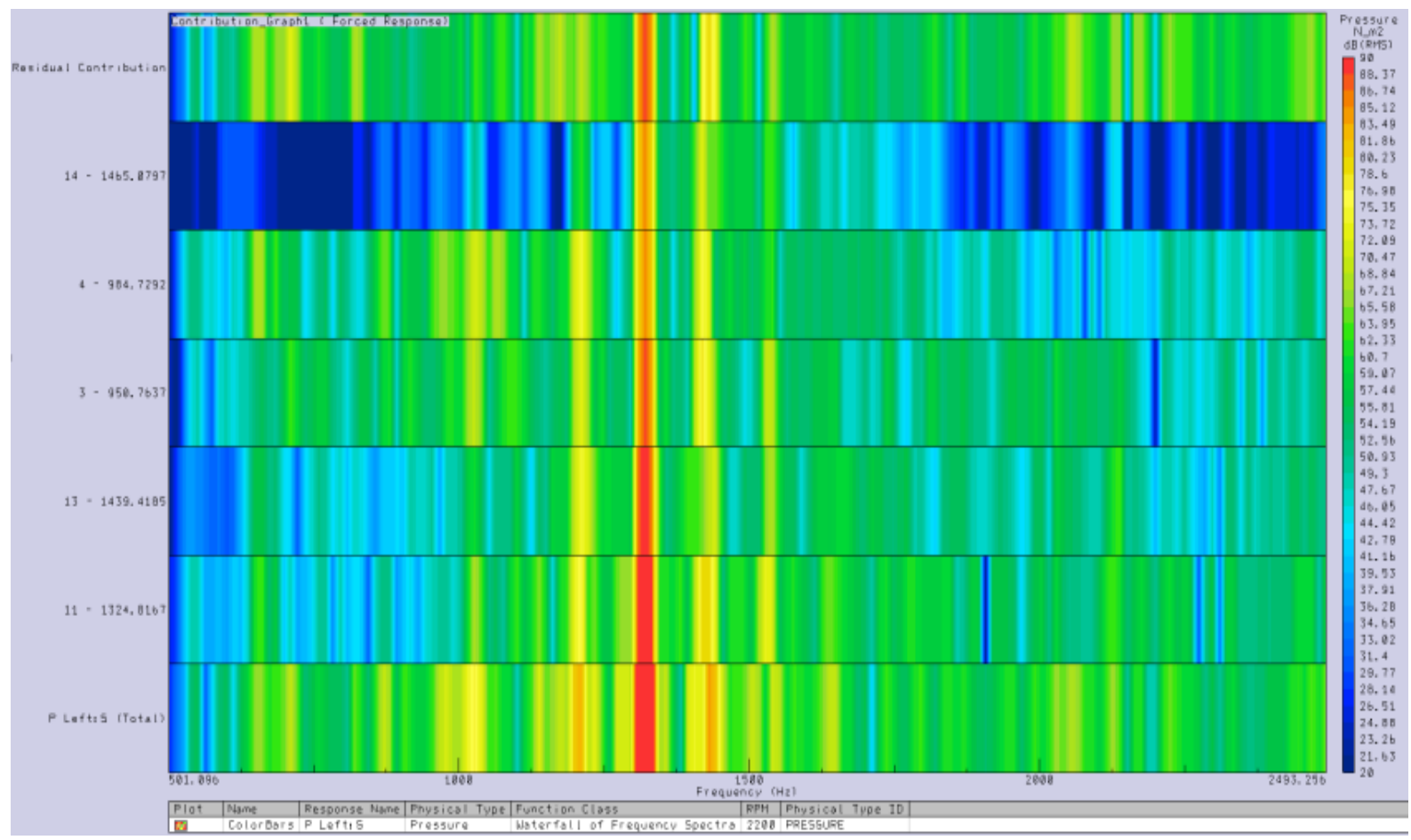

b) Diagram of the contribution of individual mode shape to the integral sound pressure level for different eigen frequencies and forms.

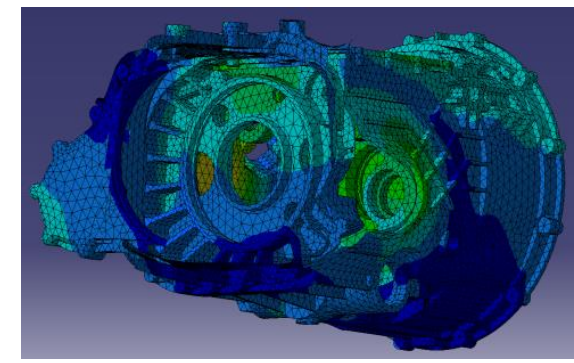

$950 \mathrm{~Hz}$

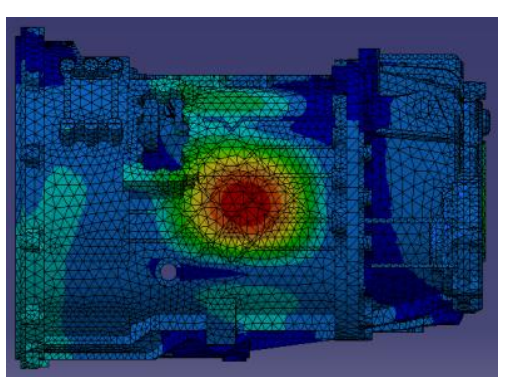

$1324 \mathrm{~Hz}$

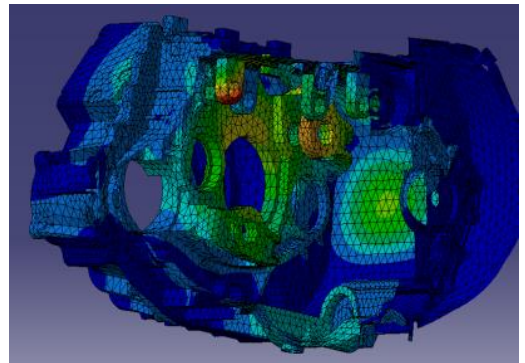

$1439 \mathrm{~Hz}$

c) Eigen forms, which make the greatest contribution to the integral sound pressure level.

Fig. 4. Waterfall diagram, diagram of the contribution of vibration eigen frequencies and the corresponding eigen forms of the transmission under study. 
points of support and equals to radial stiffness - $3 \mathrm{e} 9 \mathrm{~N} / \mathrm{m}$; axial stiffness- $5 \mathrm{e} 7 \mathrm{~N} / \mathrm{m}$; angular stiffness - 4e 5 $\mathrm{N}^{*} \mathrm{~m} / \mathrm{rad}$.

The damping of the supports is the same for all points of support: the radial damping is $10,000 \mathrm{~kg} / \mathrm{s}$; axial damping is $10,000 \mathrm{~kg} / \mathrm{s}$; corner damping $-10,000$ $\mathrm{m}^{2} * \mathrm{~kg} / \mathrm{s} / \mathrm{rad}$.

According to the available information about the loads at the points of shaft support and the dynamic stiffness of the body, described by eigen oscillation modes, the forced response in the time domain is calculated. The result of the calculation is the values of the form participation factors at any specific time, as well as the magnitudes of the movements, velocities and accelerations of each node of the finite element model of the hull, which can be visualized.

For further application of the results in acoustics calculation, a transition was made to the frequency domain with the selection of modes with constant rotation speed from a continuous signal. As a result, a set of functions of modal participation coefficients was obtained depending on the frequency and rotation speed of the input shaft.

For each of the calculated functions, a graph of frequency dependence and a waterfall diagram can be plotted, showing the dependence of the vibration shape participation coefficient value on the frequency and rotational speed of the input shaft.

\section{Optimization of configuration vibroacoustic parameters}

The acoustic mesh is built on the basis of a mechanical finite element model by constructing an envelope surface and dividing it into flat finite elements Quad4 or Tria3. The increment of the acoustic mesh is $7 \mathrm{~mm}$ and is selected for calculation within the frequency range up to $8 \mathrm{kHz}$. The waveform of the body are projected on the resulting acoustic mesh by finding the matching nodes of the meshes.

To increase the efficiency of calculation, the MATV (Modal Acoustic Transfer Vectors) method was chosen, which allows you to quickly find the value of the acoustic response at the desired point through the transfer functions calculated between all the nodes of the source model and the nodes of the receiver model. As for the response points under study, the points were selected at a distance of 0.5 meters from the side walls of the transmission align the center of the side projection.

According to the results of previous calculations, the acoustic response is calculated at the desired points for each rotational speed of the input shaft (Fig. 4a). The findings can be presented in the form of a continuous diagram, where the frequency of the acoustic response is plotted along the horizontal axis, the input shaft turns are shown along the vertical axis, and the color characterizes the amplitude of the acoustic pressure.

Based on the findings, the contribution of individual vibration modes to the integral sound pressure level was determined for different eigen frequencies and shapes (Fig. 4b). To determine the priority of corrective actions to change the configuration, a method is proposed for calculating the integral contribution of the shape to the total noise, taking into account all the considered modes.

As a key parameter of the objective function (integral noise level), the significance of the considered mode shape was estimated according to a five-point scale for different engine speeds and different gears. The maximum value of total score for the $n$-th mode shape determined the need for making configuration changes to suppress the contribution of this mode shapes in the total integral noise level.

For example, for the gearbox at the moment of 2700 $\mathrm{Nm}$, the first 50 mode shapes within the frequency range up to $8 \mathrm{kHz}$ were determined. The performed analysis based on the developed methodology allowed us to conclude that the greatest contribution to the integral noise level is made by mode shapes (Fig. 4c): at a frequency of $950 \mathrm{~Hz}$, the internal partitions of the gearbox housing; at a frequency of $1324 \mathrm{~Hz}$, the side walls of the shaft part of the gearbox and at a frequency of $1439 \mathrm{~Hz}$ are connected oscillations of the side walls and partitions between the shaft part of the gearbox and the demultiplier.

To suppress these vibrations, additional finning of the partition between the shaft part of the automatic gearbox and the demultiplier was installed, as well diagonal stiffening ribs were introduced into the configuration of the side walls of the automatic gearbox and the distributor cap. The measures taken allowed for reducing the integrated noise level by $3.5 \mathrm{dBA}$.

\section{Conclusion}

Tools for vibroacoustic parameters calculated prediction are a prerequisite for efficient transmission design engineering (noise and vibration optimization), taking into account costs and time. The application of virtual prototypes reduces transmission optimization time in combination with significantly increased quality of the first prototype. Using this methodology, modeling strategy and CAE tools in the process of optimizing vibroacoustic parameters, the prerequisites are created for solving the problems of accurate noise prediction during development of a virtual sample. Depending on the stage of transmission optimization, the level of detail (resolution) can be adapted to find the best compromise between modeling costs and modeling accuracy. For this purpose, new constructive solutions are being developed and documented.

The components, such as side surfaces, the partition between the shaft part of the automatic gearbox and the demultiplier, the distributor cap are optimized. Stiffness was increased by adding ribs in the weakest areas. The resonant frequencies are shifted to a higher frequency range, and the total sound emission power is reduced from these components. In the future, it is possible to consider application of visco-elastic materials to suppress acoustic noise. Thus, the use of all the tools presented in the virtual optimization process plays an important role in implementation of a "low-noise transmission". With regard to the object of research, the 
predicted reduction in the integral noise level is expected to be $3.5 \mathrm{dBA}$.

All the results presented above are part of current research, the purpose of which is to optimize the design of the vehicle transmission prototype.

The scientific work is carried out under the Presidential Program for Young Scientists- (Grant MK-5809.2018.8), as well as with partial funding in the framework of the state assignment of IMASH of UB RAS No. 0391 - 2014-007.

\section{References}

1. V. Derzhanskii, I. Taratorkin, S, Shelomkov, Automotive industry, Prediction of dynamic loading pre-torque converter zone HMT, № 11, pp. 32-34 (2008)

2. S. M. Jadhav, SAE Technical Papers, Powertrain NVH Analysis Including Clutch and Gear Dynamics, 2014-01-1680 (2014)

3. P. K. Singh, S.A. Raman K, SAE Technical Papers, Study of Effect of Variation in Micro-Geometry of Gear Pair on Noise Level at Transmission, 201526-0130 (2015)

4. Z. Neusser, M. Sopouch, T. Schaffner and H. Priebsch, SAE Technical Papers, Multi-body Dynamics Based Gear Mesh Models for Prediction of Gear Dynamics and Transmission Error, 201001-0897 (2010)

5. C. Chung, G. Steyer, T.Abe, M. Clapper, C. Shah, SAE Technical Papers, Gear Noise Reduction through Transmission Error Control and Gear Blank Dynamic Tuning, 1999-01-1766 (1999)

6. https://downloadly.win/siemens-lms-virtual-lab-138-x64-documentation/ Electronic resource.

7. Y. Cai J. Mech. Des 117(3), 460-469 (1995) doi: $10.1115 / 1.2826701$.

8. T. Abe, M. J. Felice, Driving the next generation of Powertrain NVH Refinement through Virtual Design, Ford Motor Company, ISMA, Leuven, (2010).

9. AUTOMOTIVE INDUSTRY STANDARD, Driver-Perceived Noise Level of Agricultural Tractors - Method of Measurement, REGD. NO. D. L.-33004/99.

10. D. Crolla, Automotive Engineering Book, ISBN: 978-0-470-97402- 5. 4101.

11. T. Reinhart, Diesel Engine Noise Control Webinar, Course ID WB1041.

12. C. Nussmann, C. Steffens, M. Atzler, Modern Tools and Methods for Low Noise Engine Development, FEV, ISMA, Leuven, (2010). 